\title{
Day-ahead Coordinated Operation of Utility-Scale Electricity and Natural Gas Networks Considering Demand Response Based Virtual Power Plants
}

\author{
Hantao Cui, Fangxing Li*, Qinran Hu, Linquan Bai, Xin Fang \\ Department of EECS, The University of Tennessee, Knoxville, TN 37996, USA
}

\begin{abstract}
The steady-state coordinated operation of electricity networks and natural gas networks to maximize profits is investigated under market paradigm considering demand response. The components in its gas supply networks are modeled and linearized under steady-state operating conditions where combined cycle gas turbine (CCGT) generators consume natural gas and bid into the electricity market. Interruptible-load based and coupon-based demand response virtual power plants are considered trading in the market like physical generators. A bi-level programming optimization model is formulated with its upper-level representing the coordinated operation to maximize profits and its lower-level simulating the day-ahead market clearing process. This bi-level problem is formulated as a mathematical program with equilibrium constraints, and is linearized as a mixed-integer programming problem. Case studies on a 6-bus power system with a 7-node natural gas system and an IEEE 118-bus power system with a 14-node gas system verifies the effectiveness of the coordinated operation model. The impacts of demand response based virtual power plants on the interactions between the two networks are also analyzed.
\end{abstract}

Keywords: Energy supply networks, Demand response, Virtual power plant, Strategic Operation, Mathematical programming with equilibrium constraints (MPEC)

\section{Introduction}

Electricity generation comes from a mix of various primary energy resources such as coal, natural gas, nuclear energy, and renewables. Combined cycle gas turbines (CCGTs), which convert chemical energy to electric energy, are widely used due to their outstanding cost-effectiveness, low $N O_{x}$ and $S O_{x}$ emissions, and quick responses. According to the Annual Energy Outlook 2014 from US Energy Information Administration [1], the demand for natural gas in the power sector in 2013 is 232.22 billion cubic meters $(\mathrm{Bcm})$. This amount is projected to rise to $266.21 \mathrm{Bcm}$ by 2040 in the reference case in which the gas delivery price is assumed to be stable. Meanwhile, industry gas demand has been growing steadily throughout the decade. With CCGT units being the largest natural gas consumers outside the industry sector, electricity transmission systems and natural gas supply networks are more closely tied and are beginning to undergo new transformations.

The linkage of CCGT units between the electricity networks and natural gas networks in a utility company affect the supply and demand balance of both networks. On the gas network side, the fluctuations of industrial and residential demand may introduce volatility in the gas supply to CCGT units and consequently affect the daily scheduling. On the electricity network side, demand response programs aiming at reducing the electricity consumption at critical high load hours also affect

*Corresponding author. Tel.: +1 8659748401
Email address: fli6@utk. edu (Fangxing Li) the power output and the gas consumption of CCGTs, which are usually the marginal generating units at load peak hours. Hence, opportunities exist for the utility company to operate the integrated energy system in a coordinated way such that the overall scheduling is optimized.

In this paper, we consider a utility company that operates an integrated energy system that consists of a power plant and a gas network. The power plant consists of CCGT units and demand response based virtual power plants. The gas network contains gas wells at the supply side and residential, industrial and CCGT units on its demand side. In a broader range, this company must strategically offer its generation at a price (or following a piece-wise price curve) to independent system operator (ISO) or a regional transmission operator (RTO). The market operator clears the day-ahead market generation offers and load bids on a social welfare maximization basis. As for the money flow, this company pays for natural gas purchases and receives payments at the rate of locational marginal pricing (LMP) for each megawatt-hour generation output.

This paper focuses on the steady-state coordinated operation of the integrated energy system in an electricity market scheme, while taking into account the impacts of demand response based virtual power plants. We formulate a bi-level optimization programming model that describes the utility company's motivation to maximize its profit by participating in the electricity market at the upper-level and simulates the market clearing process performed by the system operator at the lower level.

At the electricity network side, the demand response based 


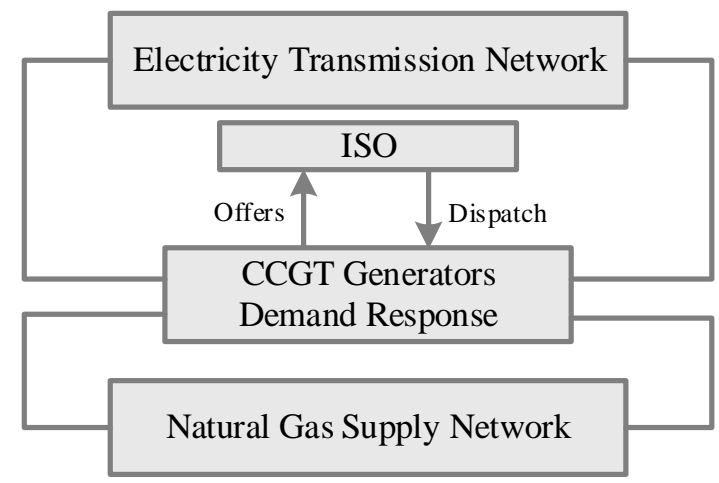

Figure 1: Structure of the Coordinated Electricity and Gas Networks

virtual power plant (VPP) behaves in the same way as the CCGT units and offers to the market at an optimum price. The offering prices of other generators as well as the total load level are obtained from forecasting. We recast the lower-level problem as its Kuhn-Karash-Tucker (KKT) optimality condition, and plugged it into the upper-level problem to form a mathematical programming with equilibrium constraints (MPEC). This model is linearized with the non-linear terms replaced with primal and dual variables, and is re-formulated as a mixed-integer programming problem (MILP).

At the natural gas supply network side, gas wells, pipelines and compressors under steady-state operation are modeled. Weymouth equation [2] is adopted to describe the natural gas flow through the pipelines, while the compressor model is also represented by its compression ratio and its power consumption. Due to the fact that the Weymouth equation and the compressor flow equation are both non-linear, they would become extremely slow to solve with the integer variables from the electricity network model. Therefore, adequate linearization techniques are utilized to maintain the linearity of the model with satisfactory accuracy.

The rest of this paper is organized as follows. Section 2 reviews the steady-state operation of gas networks and electricity works. Section 3 lays out the bi-level day-ahead coordinated operation problem, presents the linearization of the natural gas network constraints, and discusses the assumptions and models. Section 4 describes a probability model for the couponbased VPP and a deterministic model for the interruptible load VPP. Section 5 provides the mathematical solution to the MPEC model and the inclusion of VPP probability. Section 6 presents numerical studies of the model on a small test case and a larger one. Section 6 concludes the paper and outlines future research.

\section{Literature Review}

Electric power systems have been under competitive market operation with transmission network modeled over a decade ago $[3,4]$. In recent years, the study of gas network along with transmission network has gained interests among the power community [5, 6, 7]. In [5], a DC power flow model and a detailed gas network model are used for the expansion planning of combined gas and electricity network. [6] proposes a longterm co-optimization planning model of electricity and natural gas transportation infrastructures under security constraints. A multi-area and multi-stage model for expansion planning of combined electricity and natural gas network is introduced in [7] with the considerations of natural gas flow limits.

For the optimal operation of the gas-electricity network, the studies on single-time and multiple-period operational optimization of the combined gas and electricity networks are investigated in $[8,9,10,11,12,13,14,5,15,16,17]$. For a single time period snapshot, a combined natural gas and electricity optimal power flow is presented in [8]. The steady-state nonlinear gas flow equations and $\mathrm{AC}$ power flow model are considered in the optimal flow model to optimize total social welfare. Similarly, an integrated natural gas and electricity optimal power flow is presented in [9] with the objective of minimizing the sum of generating and gas supply costs. Multi-time period optimal operation is discussed in [10, 11, 12]. A multitime period combined gas and electricity network optimization model is developed and demonstrated on the Great Britain network in [11]. A multi-period generalized network flow model of the U.S. integrated energy system is presented in [12] for an integrated energy system including coal, gas and electricity network considering the economic interdependencies between the subsystems. A security based methodology is developed in [13] to solve the security constrained unit commitment problem considering the impact of the coupling natural gas networks.

Typically, in the electricity market optimization [5, 18], a DC optimal power flow model, which is linear, is adopted. However, natural gas network model is non-linear, and needs to be carefully addressed. From the perspective of the solution algorithm, most of the existing literature tends to use heuristic $[9,19]$ or non-linear [20] optimization techniques to solve the combined gas-electricity network model, which is either globally non-optimal or time consuming.

Under the deregulated market environment in electricity and natural gas networks, a utility company that supplies both electricity and natural gas [15] should account for the impact of power market and gas supply systems in the optimal operation of a combined gas-electricity network. The CCGT generators supply power to the electricity network and, meanwhile, consume natural gas provided by the gas network. According to the electricity and gas prices, the CCGT generators can decide to consume natural gas and sell electricity in the power market, or reduce natural gas consumption when the supply is insufficient [21]. Through the linkage of CCGT generators, the LMP in electricity market are interacting closely with the gas price in gas market. The impact of the natural gas network on the electricity market is of great interest to utility companies trading on markets operated by an ISO.

In recent years, demand response $[22,23,6]$ has attracted attention for its great potential and benefits to system operation. In the electricity sector, considerable work on demand response has been carried out in the electricity market and power system operation. Interruptible-load based demand response has been proven to be effective to improve system operating flexibility. The impact of price-based demand response on market clear- 
ing and the LMP in power systems has been analyzed in [23]. Only a few works considered the demand response model in the optimal operation of combined gas-electricity network. The hourly economic demand response is incorporated in the coordinated day-ahead scheduling model of power systems with natural gas network constraints [6]. None of the above take into account both of the incentive-based and price-based demand response to analyze their impacts on a coordinated system under the electricity market paradigm.

To the knowledge of the authors after extensive literature review, this is the first paper to propose stationary case day-ahead coordinated operation of gas-electric networks under an electricity market paradigm and to study the interactions between the two networks considering demand response. The novelties of this work are summarized as follows:

1. The components in natural gas networks are modeled for steady state analysis. Linearization for the pipeline flow equation and compressor flow equation are proposed;

2. A bi-level model is proposed to maximize the day-ahead profits for the utility company in the upper-level and simulate the market clearing process in the lower-level;

3. Two demand response based virtual power plant models are proposed: time-constrained IBDR-VPP and probabilistic CBDR-VPP. The parameters of CBDR-VPP are derived from survey;

4. The bi-level problem is formulated as an MPEC and transformed into an MILP by recasting the lower-level KKT conditions as constraints in the upper level problem.

\section{Bi-level Day-ahead Coordinated Operation Model}

\subsection{Modeling Assumptions}

First, we define the scope of this problem. The day-ahead coordinated operation is a steady-state scheduling problem carried out on a 24-hour time horizon with an hourly resolution. The choice of hourly resolution is to follow the electricity market offering rules, and is also sufficient to provide snapshots of steady-state natural gas networks.

Within this scope, we make the following assumptions:

1) This model is to be used by a utility company to derive hourly offering curves and schedule generation outputs for the day-ahead market, which is cleared 12-15 hours ahead;

2) The utility company pays for natural gas at a fixed rate and receives payment from the electricity market as well as natural gas consumers;

3) Unit commitment (i.e., the determination of whether a unit should be on-line or off-line) is performed before optimizing the coordinated scheduling. The unit commitment result is an input to the coordinated scheduling model;

4) Gas pipelines are isothermal and natural gas are turbulent flows. Pipelines are horizontal and only the horizontal velocity of gas is considered;

5) Transients in the natural gas networks and its components are short enough in time duration comparing with the hourly time resolution so that they are not considered.

Section 3.3 discusses the impacts of these assumptions.

\subsection{Natural Gas Network Models and Constraints}

This section provides steady-state mathematical models for natural gas network components, including CCGT units, gas pipelines, centrifugal compressors, and natural gas wells. These components are modeled to the extent of providing sufficient details to study the interactions between electricity networks and gas networks in steady-state.

\subsubsection{CCGT Units}

CCGT units are the largest industrial gas load which serve as the links between the two networks. On the gas network side, the heat needed by CCGT unit $i$ is given as a function of the generation output:

$$
F_{i, t}\left(G_{i, t}\right)=c_{i, 2} G_{i, t}^{2}+c_{i, 1} G_{i, t}+c_{i, 0}
$$

where $G_{i, t}$ is the generation output of unit $i$ at time $t$ in MW, $F_{i, t}$ is the heat consumption in $G J, c_{i, 2}, c_{i, 1}$ and $c_{i, 0}$ are the heat rate coefficients.

Costs for purchasing the natural gas is calculated based on the actual gas consumption, and the maximum gas supply to the CCGT units is limited to the contract amount:

$$
\begin{aligned}
& W_{i, t}\left(G_{i, t}\right)=\rho_{i}^{G A S} \times F_{i, t}\left(G_{i, t}\right), i \in I_{c c g t}^{N G} \\
& \sum_{i, t} F_{i, t} \leq F_{c}
\end{aligned}
$$

where $\rho_{i}^{G A S}$ is the per $G J$ cost of natural gas in US dollar, $F_{c}$ is the maximum daily fuel limit for all CCGT units in $G J$.

The efficiency of the combined cycle gas units is not explicitly used, but it is embedded in the heat rate coefficients of equation (1). Also, the standby gas consumption of CCGT units is described by the positive coefficients $c_{i, 0}$ in equation (1).

\subsubsection{Nodes and Pipelines}

The natural gas transmission system is made up of natural gas nodes and pipelines. At each time period, each node operates within the maximum and minimum pressure range:

$$
p_{n, \min } \leq p_{n, t} \leq p_{n, \max }, n \in N
$$

Natural gas flows through pipelines are dependent on the pipeline parameters and the pressure difference. For the low pressure, small diameter and short pipelines, the Weymouth equation is used to model turbulent gas flow through all pipelines at each time period:

$$
W M_{m n}\left(p_{m n}^{i n}, p_{m n}^{o u t}\right)=K_{m n}^{W} \sqrt{p_{m n}^{i n}{ }^{2}-p_{m n}^{\text {out } 2}}
$$

where $K_{m n}^{W}$ is the Weymouth factor, defined by:

$$
K_{m n}^{W}=\frac{d_{m n}^{2.67}}{K \sqrt{z_{m n} g_{m} T L_{m n}}}
$$

where $z_{m n}$ is the compressibility of the flow in pipeline $m n, g_{n}$ is the specific gravity of the flow arriving at node $m, T$ is the gas temperature, $L_{m n}$ is the length of pipeline, $d_{m n}$ is the inside 
diameter of the pipeline, and $K$ is a global constant determined by the units used [24].

In literature $[2,13,14]$ on steady-state gas network analysis, constant Weymouth factors are used by assuming the pipeline inside diameter, the gas compressibility, the temperature, and the friction factor to be constant. We adopt this assumption since constant Weymouth factors are sufficient to describe the hourly gas flow snapshots in the pipelines.

Note that the Weymouth equation is a simplified forms to describe pipeline flows. Other commonly used forms of simplified equation are Panhandle equation and Spitzglass equation. The use of simplified forms are effective provided that the appropriate form is chosen based on the pipeline characteristics. More discussions are given in Section 3.3.

\subsubsection{Gas Wells and Loads}

Gas wells are at the supply end in the natural gas network. The maximum and minimum gas production for gas wells is given as:

$$
s_{i, t}^{\min } \leq s_{i, t} \leq s_{i, t}^{\max }, i \in I_{s p}^{N G}
$$

The gas loads modeled include the industrial, commercial and residential gas consumers. The gas consumption of the CCGT units given by equation (1) and that of the compressors is also part of the gas loads on the connected node. The upper and lower limit on gas consumption is given by:

$$
d_{i, t}^{\min } \leq d_{i, t} \leq d_{i, t}^{\max }, i \in I_{\text {load }}^{N G}
$$

\subsubsection{Gas Network Nodal Balance Constraint}

Gas supplies are modeled as positive injections on the connected nodes, while gas loads are modeled as negative loads. At each time step, also enforced is the natural gas nodal balancing constraint that requires the sum of gas injections at each node to equal zero:

$$
\sum_{i \in I_{s p}^{N G}} \mathbf{A}_{s p}^{N G} s_{i, t}-\sum_{i \in I_{\text {load }}^{N G}} \mathbf{A}_{\text {load }}^{N G} d_{i, t}=\mathbf{p}_{n, t}=0
$$

\subsubsection{Centrifugal Compressors}

Compressors are installed in the gas network to transport gas by increasing the pressure that is needed to overcome the friction in pipelines. The prime-mover of the compressor consumes energy to increase the pressure at the outlet node. Compression ratio is defined as outlet node pressure over the inlet node. For each period, it is bounded within the working range:

$$
R_{j, \min } \leq R_{j, t}=\frac{p_{n}}{p_{m}} \leq R_{j, \max },\{m, n\} \in I_{c o m p}^{N G}(j)
$$

The compressor flow is a function of compressor power demand and compression ratio:

$$
f_{c, j, t}\left(H_{j, t}, p_{m, t}, p_{n, t}\right)=-\frac{H_{j, t}}{k_{2}-k_{1}\left(R_{j, t}\right)^{\alpha_{j}}}, j \in I_{c o m p}^{N G}
$$

where $H_{j, t}$ is the power consumption of compressor $j, \alpha_{j}$ is a constant set by the polytropic exponent of the compressor, and $k_{1}$ and $k_{2}$ are empirical parameters associated with the compressor design. For each period, each compressor flow is limited to its maximum permissible flow rate:

$$
f_{c, j, t} \leq f_{c, j, \max }
$$

Gas consumption of the compressors is added to the node to which the compressor inlet is connected. Extra energy consumption by compressors due to the variation of operation conditions, such as the changes in temperature and gas characteristic, may occur and increase the nodal gas load, which is not considered in this paper.

\subsubsection{Linearization of Pipeline and Compressor Models}

Both equations (5) and (11) introduce non-linearity and nonconvexity to the model, making it time-consuming to solve and impossible to find a global optimum. Equation (13) provides the first-order Taylor expansion of the non-linear Weymouth equation (5) at the given values $P I$ and $P O$, in terms of pipeline $j$ connecting nodes $m$ and $n$.

$$
\begin{aligned}
W M_{m n}\left(p_{m n}^{i n}, p_{m n}^{\text {out }}\right) & \leq W M_{m n}(P I, P O)+\frac{\partial W M_{m n}}{\partial p_{m n}^{i m}}\left(p_{m n}^{\text {in }}-P I\right) \\
& +\frac{\partial W M_{m n}}{\partial p_{m n}^{\text {out }}}\left(p_{m n}^{\text {out }}-P O\right), n \in \mathrm{N}, m \in A(n)
\end{aligned}
$$

Introduce a set of points $\left(P I_{l}, P O_{l}\right)$ to linearize the Taylor expansion (13), where $l=1,2, \ldots, L$ is the index of the point. Then replace the nonlinear Weymouth equation (5) with a set of linearized inequality constraints below:

$$
\begin{gathered}
W M_{m n} \leq K_{m n}^{W} \frac{P I_{l}}{\sqrt{P I_{m n l}^{2}-P O_{m n l}^{2}}} p_{m n}^{i n}-K_{m n}^{W} \frac{P O_{l}}{\sqrt{P I_{m n l}^{2}-P O_{m n l}^{2}}} p_{m n}^{\text {out }}, \\
n \in \mathrm{N}, m \in A(n)
\end{gathered}
$$

where for each pipeline $m n$, only one of the $L$ inequality constraints having the best approximation will be binding [2].

The gas flow of pressurized pipes is also linearized with its first-order Taylor expansion at a fixed point $\left(H_{j 0}, \pi_{m, 0}, \pi_{n, 0}\right)$. Also make sure that the initial compression ratio $R_{0}=\pi_{n, 0} / \pi_{m, 0}$ is within the limits of the compressor. The linearized formulation of (11) is given as:

$$
\begin{aligned}
f_{c, j, t}= & -\frac{H_{j 0}}{k_{2}-k_{1} R_{0}^{\alpha_{j}}}+\frac{\partial f_{c, j}}{\partial H_{j}} \times\left(H_{j, t}-H_{j 0}\right) \\
& +\frac{\partial f_{c, j}}{\partial \pi_{m}} \times\left(\pi_{m, t}-\pi_{m 0}\right)+\frac{\partial f_{c, j}}{\partial \pi_{n}} \times\left(\pi_{n, t}-\pi_{n 0}\right)
\end{aligned}
$$

where the partial derivatives can be found in Appendix B of [13]. A 50,000-sample Monte-Carlo simulation shows a maximum approximation error of $0.5 \%$ of the accurate value, which is considered to be satisfactory.

\subsection{Discussions on Assumptions and Models}

To model the interactions between gas networks and electric networks in steady-state operations, assumptions are made and simplified component models are adopted. Applicability of the assumptions and the impacts of the simplified models will be discussed in this section.

Following the industry practice in electricity markets such as the PJM ISO [25] and the New York ISO [26], the day-ahead electricity market is assumed to be clearly 12-15 hours ahead. 
Then, unit commitment of is assumed to be optimized before the coordinated scheduling so that the on/off state of units are given based on generation costs, start-up and shut-down costs, maximum on/off time constraints, and planned outages. The decisions on the on/off states represented by binary variables are then passed to the coordinated scheduling program which optimizes the scheduling of units based on fuel costs only.

The proposed coordinated operation model holds for all unit commitment inputs. Unit start-ups or shut-downs within the 24-hour period are allowed. The output power of units will be set to zero during their off-line hours and bound between the range during their on-line ones. In the objective function, the economic scheduling models do not include the unit start-up and shut-down costs since they are already taken into account in the unit commitment $[13,25]$.

On the gas network side, the pipeline is assumed to be isothermal and horizontal. Gas velocity along the horizontal velocity is assumed to be constant. Gas flow through a pipeline is originally described by the simplified equation having the same form of (5) but with a different coefficient $K_{m n}^{S}$ :

$$
K_{m n}^{S}=\frac{d_{m n}^{5 / 2}}{K \sqrt{z_{m n} g_{m} T f_{m n} L_{m n}}}
$$

where $f_{m n}$ is the (Darcy-Weisbach) friction factor in the pipeline, which is a function of the Reynolds number and the roughness of the internal relative roughness (the absolute roughness of the internal surface over the internal diameter). We will derive the Weymouth factor from this equation by approximating the Darcy-Weisbach friction factor.

The Reynolds number of gases, given by (17), is a dimension-less parameter to characterize the degree of turbulence in gas flow:

$$
\operatorname{Re}=K_{r} \times \frac{Q_{g} S}{d \mu}
$$

where $Q_{g}$ is the gas flow rate, $S$ is the specific gravity of gas at standard conditions relative to air, $d$ is the pipeline inside diameter, ${ }_{m} u$ is the gas viscosity, and $K_{r}$ is the coefficient associated with the unit used. In our case, we assume the natural gas to have a high Reynolds number $(\operatorname{Re}>4,000)$, and the gas pipeline to have smaller diameter $(d<30 \mathrm{~cm})$ and shorter length $(L<30 \mathrm{~km})$. Therefore, the friction factor is given by:

$$
\frac{1}{\sqrt{f}_{m n}}=K_{f} \times d_{m n}^{\frac{1}{6}}
$$

where $K_{f}=10.3196$ in SI units [27]. Substitute (18) into (16) yields the Weymouth factor given by (6).

The Weymouth equation is most frequently used in gas distribution networks in spite of being less exact. According to [28], this equation overestimates the pressure drop calculation. It impacts our model by underestimating the pipeline transfer capabilities and therefore making the coordinated operation more conservative in calculating the pipeline flows. When our solution is applied to an actual system, it will remain feasible and provide margins for operation.
Other than Weymouth equation for the assumed pipeline type and operating condition, the Panhandle equation and the Spitzglass equation are effective approximations in other conditions. For gas pipelines with larger diameter or long runs such as cross-country transmission pipelines for moderate Reynolds number gases, the Panhandle equation is recommended. In the low pressure vent lines, the Spitzglass equation is a more accurate approximation. An exhaustive discussion on equations for steady state flow in gas pipelines is given in [27].

\subsection{Bi-level Model}

For the utility company that owns CCGT units, conventional thermal generators and virtual power plants, we optimize its day-ahead power output scheduling. Let the CCGT units and thermal generators be the strategic ones (denoted by the superscript $S$ ) and generators owned by other participants be nonstrategic (denoted by the superscript $\mathrm{O}$ ). The problem to identify optimal operation strategy for the utility company is formulated as the following bi-level optimization model:

$$
\max \sum_{i, t} \pi_{i, t}\left(G_{i, t}^{S}+G_{i, t}^{V}\right)-\sum_{i, t}\left(W_{i, t}\left(G_{i, t}^{S}\right)+\kappa_{i, t} G_{i, t}^{V}\right)
$$

subject to:

$$
-R_{i}^{D N} \leq G_{i, t+1}^{S}-G_{i, t}^{S} \leq R_{i}^{U P}
$$

Natural gas network contraints (3) - (13)

$$
\begin{aligned}
& \left\{G_{i, t}^{S}, G_{i, t}^{V}, \pi_{i, t}\right\} \in \arg \left\{\begin{array}{c}
\text { Minimize } \sum_{i, t} \alpha_{i}^{S} G_{i, t}^{S} \\
\quad+\sum_{i, t} \alpha_{i}^{V} G_{i, t}^{V}+\sum_{i, t} \alpha_{i}^{O} G_{i, t}^{O}
\end{array}\right. \\
& \sum_{i}\left(G_{i, t}^{S}+G_{i, t}^{V}+G_{i, t}^{O}\right)=\sum_{i} D_{i, t}: \lambda_{t}, \forall t \\
& G_{i, \text { min }}^{S} \leq G_{i, t}^{S} \leq G_{i, \max }^{S}: \omega_{i, t, \min }^{S}, \omega_{i, t, \max }^{S}, \forall i \forall t \\
& G_{i, \text { min }}^{O} \leq G_{i, t}^{O} \leq G_{i, \text { max }}^{O}: \omega_{i, t, \min }^{O}, \omega_{i, t, \max }^{O}, \forall i \forall t \\
& 0 \leq G_{i, t}^{V} \leq G_{i, \max }^{V}: \omega_{i, t, \min }^{V}, \omega_{i, t, \max }^{V}, \forall i \forall t \\
& -C_{l} \leq \sum_{i} G S F_{l-i} \times\left(G_{i, t}^{S}+G_{i, t}^{O}+G_{i, t}^{V}-D_{i, t}\right) \leq C_{l}: \\
& \mu_{l, t}^{\min }, \mu_{l, t}^{\max }, \forall t, \forall l \\
& \left.\pi_{i, t}=\lambda_{t}+\sum_{i} G S F_{l-i}\left(\mu_{l, t}^{\min }-\mu_{l, t}^{\max }\right)\right\}
\end{aligned}
$$

The objective of the upper level (19) maximizes the daily profit of the utility company that buys natural gas and generates electricity. (20) limits the hourly output ramping for the strategic CCGT units. The objective function of the lower level problem (22) simulates the market clearing process performed by an ISO which minimizes the total cost for purchasing energy from strategic, conventional and virtual generators. (23) enforces the power balancing constraint for each time interval. Constraints (24)-(26) bind the maximum and minimum output for strategic, ordinary, and virtual generators. (27) constrains the power flow through each transmission line. (28) derives the LMP for each bus. The notations on the right-hand-side of the colons of each lower-level constraint are the associated dual variables. 


\section{Modeling Virtual Power Plants}

\subsection{Virtual Power Plant from Demand Response}

Virtual power plant (VPP), by definition, is an electric power generation system that can integrate several types of small to medium scale generations, such as micro combined heat and power (uCHP) systems, wind turbines, PV, and batteries. The inclusion of demand-side management further extends the scope of a virtual power plant. With a sophisticated central energy management system (EMS), those different sources are coordinated to provide reliable power generation like a conventional power plant.

DR is a considerable source of virtual power plant capacity $[29,30]$. DR programs are categorized into two major types according to the response mechanism: incentive-based and price based. Participants of incentive-based DR programs, such as direct load control, demand bid/buy-back and capacity programs, usually have a higher demand comparing to the pricebased DR customers. They sign contracts with utility companies to receive payments for load curtailment, or directly bid and buy from the electricity market. On the other hand, pricebased, small capacity DR participants are mostly residential customers, who voluntarily reduce power consumption during higher price periods.

In this section, VPPs are modeled by demand response resources. Two types of DR programs, i.e coupon-based DR (CBDR) and interruptible-load based DR (IBDR), are considered. CBDR is a subclass of price-based DR in which customers obtain the offered rebates by reducing load voluntarily. IBDR is a subclass of incentive-based DR in which customers get compensated from electricity use interruptions. Note that although this paper only proposes DR models for VPP, a similar modeling approach can be taken to include conventional or renewable energy resources for VPP.

\subsection{Coupon-based Demand Response VPP Model}

To describe the characteristics of coupon based demand response (CBDR), the model focuses on the probability of load reduction level as functions of incentive time, level and load type. Such characteristics are sampled from the real world to reflect the preferences of users.

To obtain the distribution of response probabilities for VPP modeling, a six-step procedure is proposed in [18] which involves three surveys: 1. Residential Energy Consumption Survey (RECS) by Energy Information Administration (EIA), 2. American Time of Use Survey (ATUS) by Department of Labor, and 3. a consumer-reported DR behavior survey (DRBS) conducted by Center for Ultra-wide-area Resilient Electric Energy Transmission Network (CURENT). The DRBS survey collected 177 U.S. residents across 48 states to study consumers' reactions to financial incentives by estimating the adoption rates of major DR programs as a function of the financial rewards.

As an example, the estimated probability distribution of power reduction in a certain area for a certain time is given. The impact of the incentive level on load reduction from actual data is shown in Fig. 2 [18]. For each load level, the load reduction

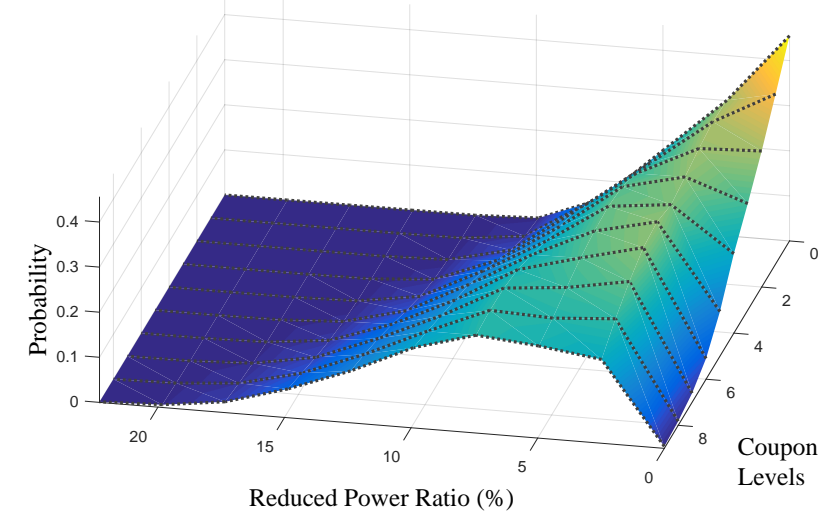

Figure 2: Probability Distribution of Load Reductions Following the Coupon Level

ranges from zero to $25 \%$ unimodally. The mean load reduction increases along with the incentive level. This probability distribution of CBDR VPP will be included in the coordinated operation model by different scenarios. The scenario-based approach will be given in Section 5.3.

\subsection{Interruptible-load based Demand Response VPP Model}

Compared to the CBDR, the interruptible-load based DR (IBDR) is more deterministic from the perspective of availability. The contracts with IBDR customers allow the utility company to interrupt the load at the contract price $\kappa_{j}$ for a maximum response time of $M U_{j}$. Also specified in the contract is the minimum time interval $M D_{j}$ between two interruptions. The enforced maximum response time and minimum interrupt interval requirements of the IBDR are modeled with a set of mixed-integer constraints expressed as:

$$
\begin{aligned}
& \sum_{t=1}^{L_{j}} v_{j, t}=0, \forall j \in J \\
& \sum_{t=k}^{k+M D_{j}-1}\left(1-v_{j, t}\right) \geq M D_{j}\left(v_{j, k-1}-v_{j, k}\right), \\
& \forall j \in J, \forall k=L_{j}+1, \ldots, T-M D_{j}+1 \\
& \sum_{t=k}^{T}\left[1-v_{j, t}-\left(v_{j, k-1}-v_{j, k}\right)\right] \geq 0, \\
& \forall j \in J, \forall k=T-M D_{j}+1, \ldots, T \\
& \left(\sum_{t=k}^{k+M U_{j}} v_{j, t}\right) \leq M U_{j} \\
& \forall j, \forall k=L_{j}+1, \ldots, T-M U_{j}
\end{aligned}
$$

Equation (29) sets the initial status for the VPP if it must be off due to previous operations. (30) requires the VPP to be offline for $M D$ consecutive hours once it is shut down. (31) in addition requires the VPP to stay off-line till the last period if the remaining hours are less than $M D$. (32) imposes the maximum on-line time constraint for IBDR VPP. 


\section{Mathematical Solution}

\subsection{Formulation of an MPEC}

$\operatorname{Max} \sum_{i, t} \pi_{i, t}\left(G_{i, t}^{S}+G_{i, t}^{V}\right)-\sum_{i, t}\left(W_{i, t}\left(G_{i, t}^{S}\right)+\kappa_{i, t} G_{i, t}^{V}\right)$

Contraints in (3) - (13) and (20)

$\alpha_{i}^{\phi}=\lambda_{t}+\left(\omega_{i, t, \min }^{\phi}-\omega_{i, t, \min }^{\phi}\right)+\sum_{i}\left[G S F_{l-i}\left(\mu_{l, t}^{\min }-\mu_{l, t}^{\max }\right)\right]$

$0 \leq \omega_{i, t, \min }^{\phi} \perp\left(G_{i, t}^{\phi}-G_{i, \min }^{\phi}\right) \geq 0$

$0 \leq \omega_{i, t, \min }^{\phi} \perp\left(G_{i, \text { max }}^{\phi}-G_{i, t}^{\phi}\right) \geq 0$

$\forall \phi \in\{S, V, O\}, \forall i, \forall t$ for $(35)-(37)$

By recasting the lower-level problem with its KKT optimality conditions (35)-(37), the bi-level optimization problem is transformed into an MPEC formulation. Non-linearlities in the both the objective and the constraints should be converted to linear ones in order to formulate a solvable MILP problem [31].

\subsection{Mixed-integer Programming Solution}

The goal of converting the MPEC into an MILP is to eliminate the nonlinearities in (33) - (37), which include:

1) the product term $\pi_{i, t}\left(G_{i, t}^{S}+G_{i, t}^{V}\right)$ in the objective function;

2) the quadratic costs $W_{i, t}(\cdot)$ in the objective function;

3 ) the complementary conditions from KKT in (34) - (37).

The complementary conditions can be linearized with the method in [32]. The linearization of 2) and 3) are stated in Appendix A and Appendix B. The final mixed-integer linear programming problem, equivalent to the MPEC in (33)-(37) is formulated as:

$$
\begin{aligned}
\text { Max } & \sum_{i, t} \pi_{i}\left(G_{i}^{S}+G_{i}^{V}\right)-\sum_{i, t}\left(W_{i, t}\left(G_{i, t}^{S}\right)+\kappa_{i, t} \times G_{i, t}^{V}\right) \\
= & \lambda \sum_{i} D_{i}-\sum_{i} \omega_{i, \max }^{O} G_{i, \max }^{O}+\sum_{i} \omega_{i, \min }^{O} G_{i, \min }^{O} \\
+ & \sum_{l} \mu_{l}^{\min }\left(-C_{l}+\sum_{i} G S F_{l-i} \times D_{i}\right) \\
+ & \sum_{l} \mu_{l}^{\max }\left(-C_{l}-\sum_{i} G S F_{l-i} \times D_{i}\right) \\
& -\sum_{i} \alpha_{i}^{O} G_{i}^{O}-\sum_{i, t} W_{i, t}^{L}\left(G_{i, t}^{S}\right)-\sum_{i, t}\left(\kappa_{i, t} \times G_{i, t}^{V}\right)
\end{aligned}
$$

subject to:

Contraints in (3) - (13), (20), (35), and (B.1) - (B.6)(40)

Contraints in $(29)-(32)$

$$
\begin{aligned}
& 0 \leq \mu_{l}^{\min } \leq M_{\mu}^{\min } v_{\mu, l}^{\min } \\
& 0 \leq C_{l}+\sum_{i, \phi} G S F_{l-i} \times\left(G_{i, t}^{\phi}-D_{i, t}\right) \leq M_{\mu}^{\min }\left(1-v_{\mu, l}^{\min }\right) \\
& 0 \leq \mu_{l}^{\max } \leq M_{\mu}^{\max } v_{\mu, l}^{\max }
\end{aligned}
$$

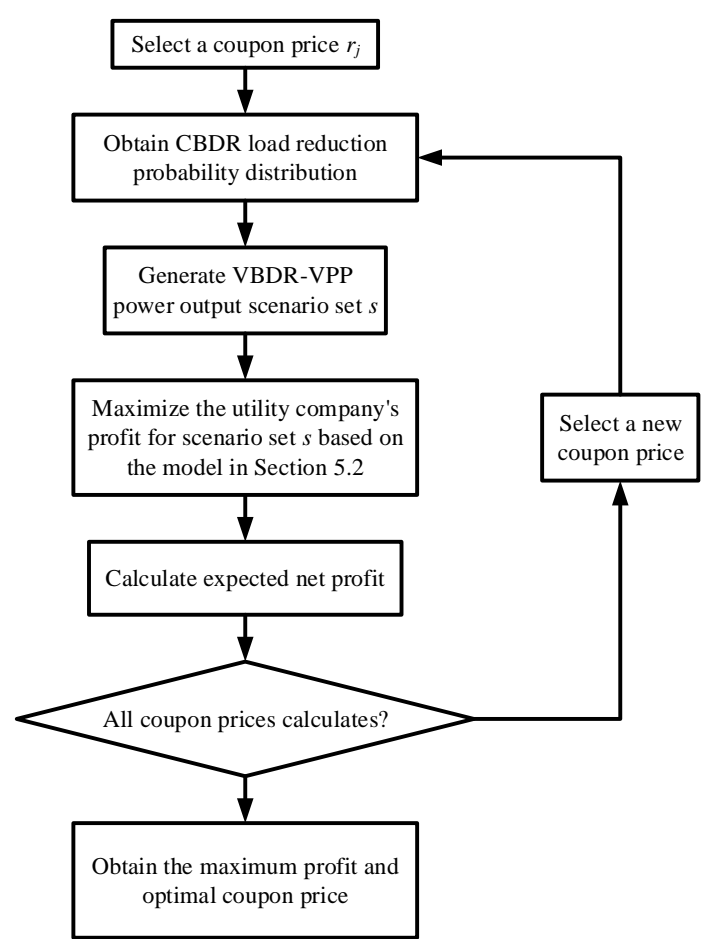

Figure 3: Solution Flowchart of DR-VPP Included Coordinated Operation Model

$$
\begin{aligned}
& 0 \leq C_{l}-\sum_{i, \phi} G S F_{l-i} \times\left(G_{i, t}^{\phi}-D_{i, t}\right) \leq M_{\mu}^{\max }\left(1-v_{\mu, l}^{\max }\right) \\
& 0 \leq \omega_{i, t, \min }^{\phi} \leq M_{\omega, \phi}^{\min } v_{\omega, i, t, \min }^{\phi} \\
& 0 \leq G_{i, t}^{\phi}-G_{i, \min }^{\phi} \leq M_{\omega, \phi}^{\min }\left(1-v_{\omega, i, t, \min }^{\phi}\right) \\
& 0 \leq \omega_{i, t, \max }^{\phi} \leq M_{\omega, \phi}^{\max } v_{\omega, i, t, \max }^{\phi} \\
& 0 \leq G_{i, \max }^{\phi}-G_{i, t}^{\phi} \leq M_{\omega, \phi}^{\max }\left(1-v_{\omega, i, t, \max }^{\phi}\right)
\end{aligned}
$$

where $M_{\mu}^{\min }, M_{\mu}^{\max }, M_{\omega, \phi}^{\min }$, and $M_{\omega, \phi}^{\max }$ are big constants, $v_{\mu, l}^{\min }$, $v_{\mu, l}^{\max }, v_{\omega, i, t, \min }^{\phi}$, and $v_{\omega, i, t, \max }^{\phi}$ are auxiliary binary variables associated with the dual variables.

\subsection{Inclusion of Probabilistic CBDR VPP Models}

In section 5.2, IBDR-VPP is included in the deterministic day-ahead coordinated operation model. However, to include CBDR-VPP characterized by probability distributions, the distributions need to be sampled to generate corresponding CBDR-VPP output scenarios before the deterministic models. The flowchart of the overall procedure is shown in Fig. 3.

$$
E N R_{r j}=\sum_{i \in S N O} p r_{i} \times R_{n, i, r j}
$$

The coupon prices are selected discretely within the surveyed coupon price range. Each coupon price is sampled to generate a group of scenarios with the same probability. For each scenario, 


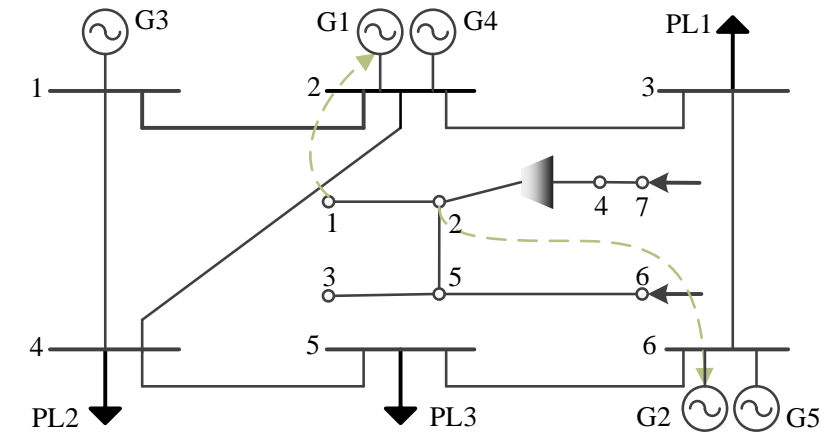

Figure 4: Six-bus system with a Seven-node Natural Gas Networks

the day-ahead scheduling optimization model is a deterministic one, and the optimal solution has the same probability as the scenario. Optimize the profit for each scenario and calculate the expected net profit with (50). Calculate recursively over each coupon price, and select the coupon price that achieves the highest profit.

\section{Case Study}

The proposed coordinated operation strategy is applied to two cases consisting of a six-bus electric system with a sevennode gas system and the IEEE 118-bus system with a 14-node gas system to illustrate its effectiveness. Assume $1 \mathrm{Mcm}$ natural gas can generate $37.26 G J$ energy in the case studies (equivalent to assume 1 cubic feet gas to generate 1 million British thermal unit energy). The coordinated scheduling is carried out on a 24-hour time horizon with a 1-hour interval, which is sufficient for gas networks and is also the current practice in day-ahead electricity markets.

\subsection{Six-Bus System}

The six-bus electric system is modified from [13]. It has five physical generators, two IBDR-VPPs, and seven transmission lines. The economic dispatch of the whole system is managed by the ISO, while the utility company that this paper examines owns and operates two out of the five generators and two IBDRVPPs according to the operational constraints or contracts. The parameters of buses, generators, branches and the 24-hour horizon load data can be found in C.7 in Appendix C. The two IBDR-VPPs are installed on buses 4 and 5 and both rated at 40 MW. For the coordinated operation study, we assume unit commitment is performed in advance and all generators are committed for the whole periods. The seven-node natural gas system consists of two suppliers, five pipelines, one compressor, one gas storage, and three residential loads. Parameters of the gas system can be found in C.9-C.14 in Appendix C.

The electric system and the natural gas system are coupled by the two CCGT units owned by the utility company. Gas loads $L_{1}$ and $L_{2}$ on nodes 1 and 2 correspond to the gas consumption of units $\mathrm{G} 1$ and $\mathrm{G} 2$, determined by the hourly generation output. Residential natural gas loads are aggregated as $L_{3}$ and $L_{4}$ on

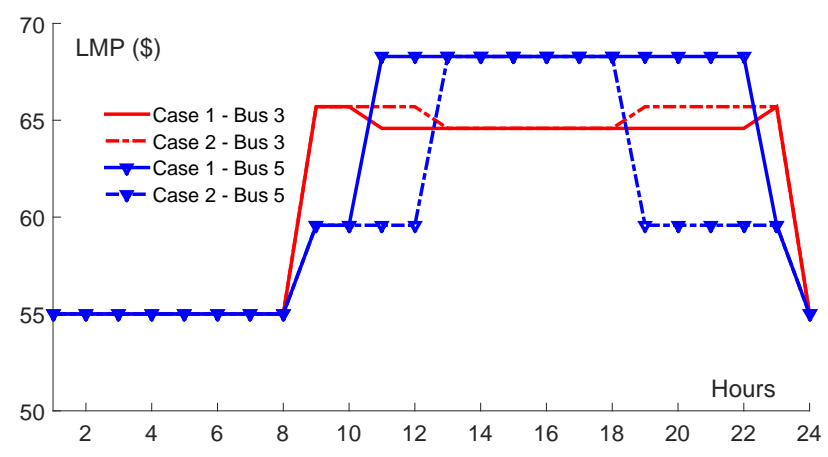

Figure 5: Locational Marginal Price of Bus 3 and 5 in Case 1 and Case 2

nodes 1 and 3. The 24-hour horizon residential gas demand is given in C. 15 .

To demonstrate the effectiveness of the proposed coordinated operation approach and illustrate the interactions between electric demand response and natural gas transmission system, four cases are considered:

1) Integrated operation without demand response

2) Integrated operation with IBDR-VPP

3) Integrated operation with IBDR and CBDR VPP

4) Impact of natural gas pipeline outages

Case 1: Integrated operation without demand response: the 24-hour operating profit is maximized with the proposed MILP model with DC power flow based line-flow constraints and gas network constraints and without any demand response based VPP. The LMP of buses 3 and 5 are shown in Fig. 5. When the electric load peaks at hour 17, units 1 to 5 are generating at $0 \mathrm{MW}, 95.75 \mathrm{MW}, 137.43 \mathrm{MW}, 10 \mathrm{MW}$ and 12.82 MW, respectively. The expensive unit 1 is not generating because it is the marginal unit at that hour, and any output change would raise the LMP and reduce the profits. Although the residential gas load peak does not coincide with the electric load peak, the total natural gas demand peaks at hour 17, and pipeline 2 transmits at its maximum capacity of $107.9 \mathrm{Mcm} / \mathrm{h}$. The optimized daily operating profit for Case 1 is $\$ 27,732$.

Case 2: Integrated operation with IBDR-VPP: Two interruptible-load based VPPs described by (29)-(32) are set on-line at buses 4 and 5 in the electric grid, while all other parameters remain unchanged. The contract price for dispatching virtual generation can be set as time varying, but for the sake of simplicity, it is assumed to be $\$ 25 / \mathrm{MW}$ for hours 1 to 12 , and \$40/MW for hours 13 to 24 , respectively. To motivate the utility company, the contract price is typically lower than the generator unit marginal costs. Table 1 shows the scheduling of IBDR-VPP which are initially off-line but dispatchable.

The LMP on buses 3 and 5 are plotted in Figure 5. At LMP critical hours, taking hour 9 to hour 12 as an example, the dispatch of VPP may affect the binding constraints in the model and therefore change the LMP. Although the dispatch of VPP does not guarantee a drop in LMP, it always counteracts the impact of increasing load on LMP. Comparing to Case 1, the generation outputs of the coordinated CCGT units remain the same 


\begin{tabular}{l|l|l|l|l|l|l}
\hline \multicolumn{7}{c}{ Daily Profit: $\$ 36,341$} \\
\hline & 1 & 2 & 3 & 4 & 5 & 6 \\
\hline 1 & 15.2 & 40.0 & 0 & 0 & 0 & 0 \\
2 & 40.0 & 5.2 & 0.0 & 0.0 & 0.0 & 0.0 \\
\hline & 7 & 8 & 9 & 10 & 11 & 12 \\
\hline 1 & 0.0 & 0.0 & 40.0 & 40.0 & 40.0 & 40.0 \\
2 & 0.0 & 0.0 & 37.7 & 40.0 & 40.0 & 40.0 \\
\hline & 13 & 14 & 15 & 16 & 17 & 18 \\
\hline 1 & 0.0 & 0.0 & 0.0 & 0.0 & 0.0 & 0.0 \\
2 & 0.0 & 0.0 & 0.0 & 0.0 & 0.0 & 0.0 \\
\hline & 19 & 20 & 21 & 22 & 23 & 24 \\
\hline 1 & 40.0 & 40.0 & 40.0 & 40.0 & 0.0 & 0.0 \\
2 & 40.0 & 40.0 & 40.0 & 40.0 & 0.0 & 0.0 \\
\hline
\end{tabular}

Table 1: Hourly IBDR-VPP Scheduling of Case 2

\begin{tabular}{l|l|l|l|l|l|l}
\hline \multicolumn{7}{c}{ New Daily Profit: \$35 196 } \\
\hline & 18 & 19 & 20 & 21 & 22 & 23 \\
\hline 1 & 0 & 40 & 40 & 40 & 40 & 0 \\
2 & 0 & 40 & 40 & 40 & 40 & 0 \\
\hline 1 & 0 & 0 & 40 & 40 & 40 & 40 \\
2 & 20.58 & 26.65 & 40 & 40 & 0 & 0 \\
\hline
\end{tabular}

Table 2: VPP Scheduling of Case 2 in 1.0x and 1.2x Residential Gas Load

at hour 17, but they are reduced from $0 \mathrm{MW}, 83.75 \mathrm{MW}, 137.43$ MW, $10 \mathrm{MW}$ and $12 \mathrm{MW}$ in Case 1 to $18.19 \mathrm{MW}, 27.78 \mathrm{MW}$, $100 \mathrm{MW}, 10 \mathrm{MW}$ and $10 \mathrm{MW}$ at hour 19 , respectively. Note that VPP does not kick in at hour 17 due to the profit maximization objective.

Comparison between the gas flow through pipeline 2 and pipeline 4 is shown in Fig. 6. Changes of gas flow through these two pipelines matter because they are connected to terminal nodes that have both residential gas demand and CCGT units. Although the VPP is off-line at hours 15-18, it does not help to reduce the pipeline flow until hour 19. This is a reasonable solution for the objective defined in the model to maximize the daily profit, as long as all the electricity transmission and gas supply network constraints are satisfied. In other words, any change in the schedule of VPP in hours 15-18 would change the schedule afterwards and reduce the profit in hours 19-22, resulting in an overall profit loss.

Other than pure profit-driven motivation, IB-VPPs are also observed to help mitigate natural gas pipeline congestion in extreme cases where the coordinated operation problem would be infeasible without IB-VPP. Multiply the residential gas demand by 1.2 throughout the day and solve Case 2 again; the VPP scheduling changes between hour 18 to hour 23 are listed in Table 2. In this case, VPP is dispatched at hours 18 and 23 to reduce the pipeline flow and avoid the infeasibility of the problem, at the cost of the reduced profit $\$ 1,145$.

Case 3: Integrated operation with IBDR-VPP and CBDR$V P P$ : The inclusion of coupon-based on top of Case 2 involves sampling from a probability distribution described in Section 4. A scenario-based approach is adopted by maximizing the expected net profit (ENP) problem consisting of a set of individ-

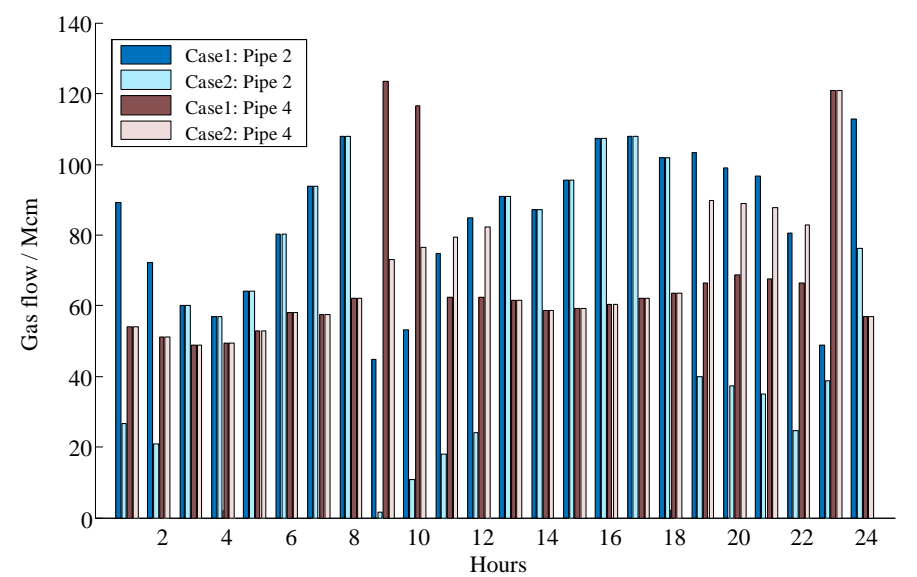

Figure 6: Gas Flow through Pipeline 2 and 4 in Case 1 and Case 2
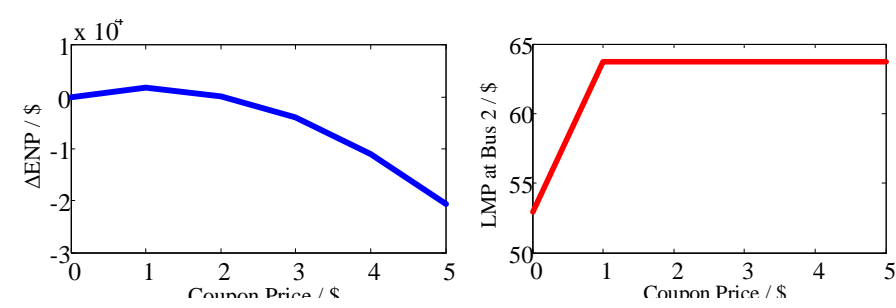

Figure 7: $\triangle$ ENP Change and Hour-19 Bus-2 LMP Change Following Coupon Price

ual problems with different scenario data inputs. Details of the scenario-based approach are omitted due to space limits but can be found in [18]. As for the parameters, 50\% of the residential electricity load is assumed to be responsive to coupons, aggregated as CBDR VPPs. The coupon price range is set from 0 to $\$ 5$ of the bus LMP and is evenly divided into 6 levels, where the coupon level 0 is equivalent to Case 2 .

As observed from the results shown in Fig. 7, the profit increases at coupon level $1(\Delta \mathrm{ENP}>0)$, falls back to the initial value at coupon level 2 , and decreases sharply after coupon level 3. The profit increase is explained by the shifted operating point that increased the LMP at bus 2, which allows the utility company to receive more payment than Case 2. The declination of profit is due to the reduced output of IBDR-VPP and CCGT units and the consequent reduced payment, as well as the unnecessary coupon costs.

Case 4: Impact of natural gas pipeline outage:

Natural gas pipeline outage is a contingency that has serious consequences in integrated energy systems with high CCGT unit capacity. Insufficient generation from CCGT units due to pipeline outage may incur high demand response dispatch and load shedding. In this case, the outage of pipeline 2 between nodes 2 and 5 at peak hours 21-24 cuts off the gas transmission from supplier 1 to gas loads on the lower branch. The comparison of pipeline flow and the output of strategic generator 2 is shown in Fig. 8.

Pipeline outage in hours 21-24 directly causes a supply shortage of natural gas on bidding generator 2 and reduces its output to a lower level, as shown in Fig. 8. To compensate for the elec- 

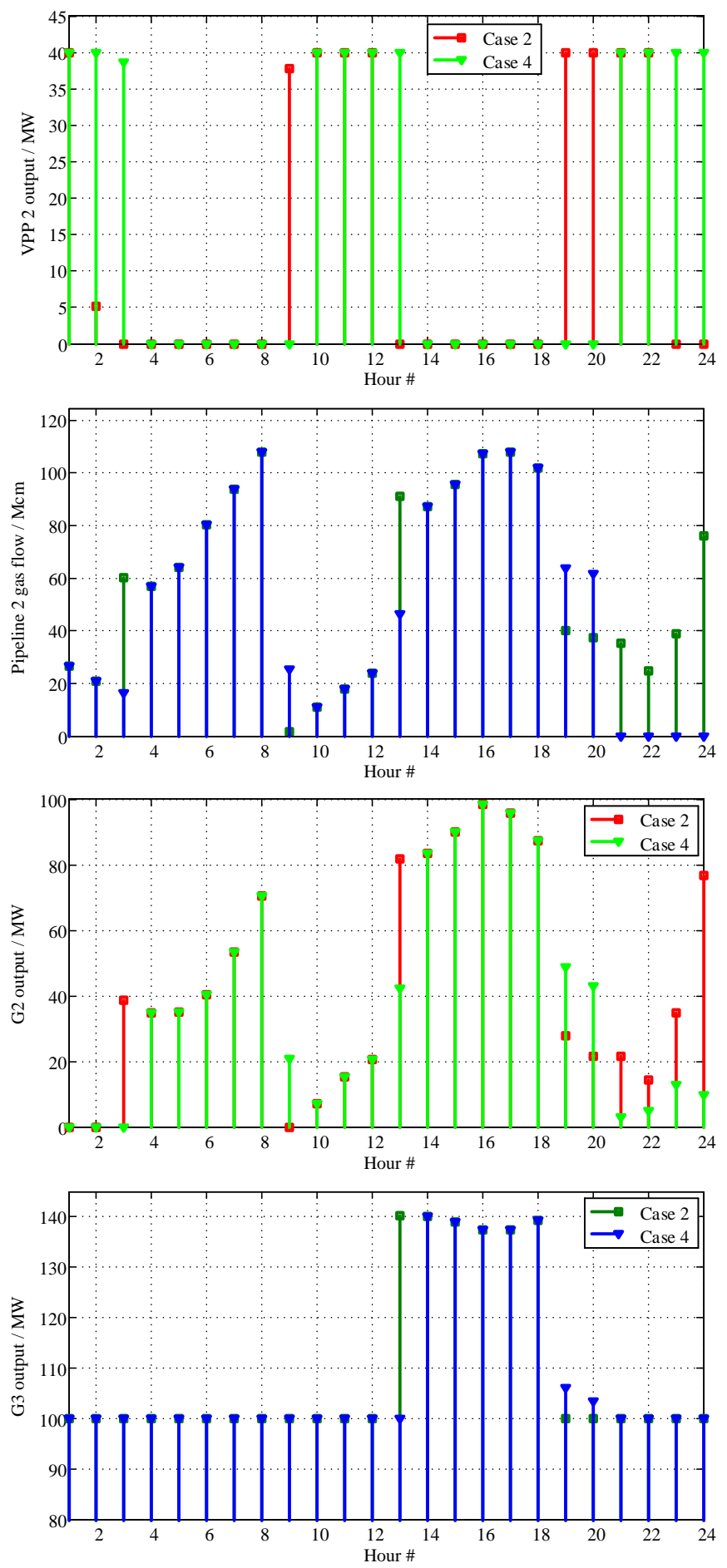

Figure 8: Comparison of Hourly System Snapshots Considering Pipeline 2 Outage

\begin{tabular}{c|c|c|c|c}
\hline Cases & no VPP & IBDR-VPP & CBDR-VPP & Both \\
\hline CL-1 & 68,917 & 74,841 & 69,600 & 75,523 \\
\hline CL-2 & - & - & 67,360 & 73,284 \\
\hline CL-3 & - & - & 62,927 & 68,850 \\
\hline CL-4 & - & - & 55,576 & 61,498 \\
\hline CL-5 & - & - & 45,790 & 51,712 \\
\hline
\end{tabular}

Table 3: Total Profit of the Utility Company with and without VPPs in Dollars

tricity supply shortage, the scheduling of IBDR-VPP is changed to on-line at hours 21-24, shown in Fig. 8(c). Fig. 8(d) plots the output of non-bidding generator 1 over the studied periods.

\subsection{IEEE 118-bus System}

In this section, a large gas and electricity integrated system consisting of a modified IEEE 118-bus system and a 14-node gas network [13] is used to demonstrate the validity of the proposed method. In the modified 118-bus system, 12 out of 73 generators are CCGT, which are owned and operated by the utility company. On buses 4 and 6, IBDR-VPP are installed and rated at $40 \mathrm{MW}$, while on buses 3, 4, and 6 CBDR-VPP are implemented. The electricity network also contains 186 branches and 91 physical loads, which peak at $7300 \mathrm{MW}$. In the natural gas supply network, 3 gas wells, 12 pipelines, 2 compressors and 5 residential gas load are included. The whole system structure is shown in Fig. 9.

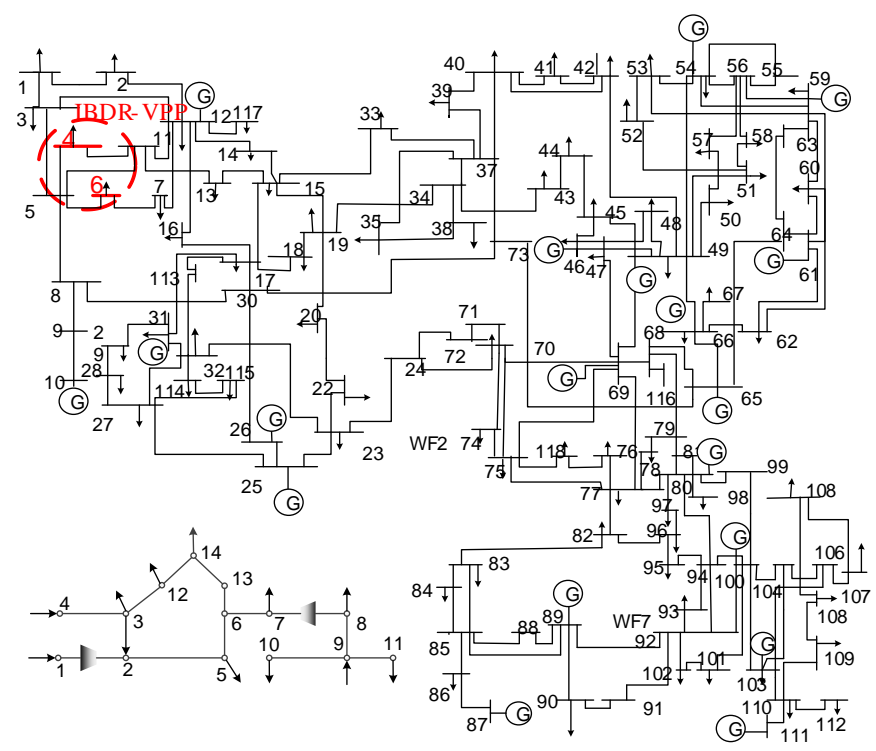

Figure 9: IEEE 118-bus System with a 14-node Gas Network

Simulation scenarios are designed in the following way: 1) a case without any demand response based VPP; 2) a case with only IBDR-VPP; 3) 5 cases with only CBDR-VPP at different coupon levels; 4) 5 cases with IBDR-VPP and CBDR-VPP at different coupon levels. It can be seen from the profit listed in Table 3 that implementation of both IBDR-VPP and CBDRVPP can gain more profit from day-ahead coordinated operation. A similar declining trend in profit from CBDR-VPP along 


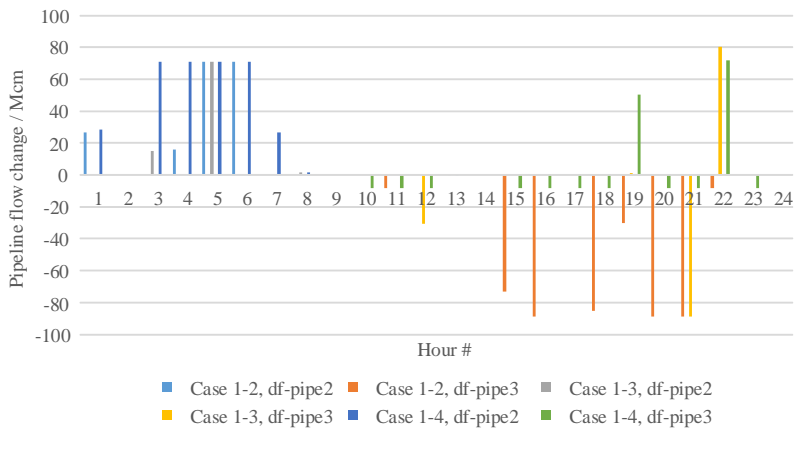

Figure 10: Change of Gas Flow in Pipelines 2 and 3 Comparing with Case 1

with the increasing coupon rebate level is also observed in the IEEE 118-bus case, implying that, for the load of the same elasticity, there exists an optimal coupon incentive to achieve the maximum profit. However, it should be noted that the optimal coupon incentive value cannot be optimized from a deterministic model as CBDR-VPP is considered by means of scenarios.

Pipeline flows in the natural gas supply system are also affected by demand response based VPPs. Let Case 1 be the base case, the changes in gas flow in pipelines 2 and 3 in Case 2 to Case 4 under coupon level 1 is depicted in Fig. 10. In this 14 node gas network with sufficient supply capability, although not significantly reducing any congestion, IBDR-VPP and CBDRVPP shifts some gas flow from pipeline 3 to pipeline 2 by reducing the output of CCGT generator 69 , and reduced the overall gas natural gas demand at peak hours. Finally, LMP in the market is only affected to the second decimal place due to the relatively small scale of this utility company.

\section{Conclusions}

This paper presents a bi-level optimization model for dayahead coordinated operation of an electricity a network and natural gas system. In this paper, the electricity market clearing process is modeled and incorporated into the coordinated operation model to formulate a MPEC model, wherein the nonlinear components are linearized and the DR is modeled as a VPP in the electricity network.

The main conclusions from case studies are:

1. The profit increase from VPP's bidding in electricity market depends on the type of VPP: IBDR-VPP can guarantee no less profit than the case without any VPP; however, CBDR-VPP may increase or reduce the overall profit depending on the coupon price level;

2. The LMP in electricity markets can see reductions with the participation of VPP. The reduction level is dependent on system size and utility scale; in the six-bus system, this effect is more significant than in the larger IEEE 118-bus system.

3. Demand response based VPP can help to reduce natural gas network congestion at peak gas demand hours at the cost of reduced profits.
Future work on this topic is to consider renewable energy integration and energy storage deployments.

\section{Acknowledgment}

The authors would like to acknowledge the support in part by the NSF grant ECCS-1001999 and the CURENT research center funded by NSF and DOE under the NSF grant EEC1041877 .

\section{References}

[1] US Energy Information Administration, Annual Energy Outlook 2015, Tech. rep. (2014).

[2] G. Hasle, K.-A. Lie, E. Quak, T. Hagen, M. Henriksen, J. Hjelmervik, Optimization Models for the Natural Gas Value Chain, in: Geometric Modelling, Numerical Simulation, and Optimization, no. December, Springer, 2007, pp. 211-264.

[3] J. Yang, F. Li, L. Freeman, A market simulation program for the standard market design and generation/transmission planning, in: 2003 IEEE PES General Meeting, 2003, pp. 442-446.

[4] H. Chao, F. Li, L. H. Trinh, J. Pan, M. Gopinathan, D. J. Pillo, Market based transmission planning considering reliability and economic performances, in: 2004 International Conference on Probabilistic Methods Applied to Power Systems, 2004, pp. 557-562.

[5] M. Chaudry, N. Jenkins, M. Qadrdan, J. Wu, Combined gas and electricity network expansion planning, Applied Energy 113 (2014) 1171-1187.

[6] X. Zhang, M. Shahidehpour, A. Alabdulwahab, A. Abusorrah, Hourly Electricity Demand Response in the Stochastic Day-Ahead Scheduling of Coordinated Electricity and Natural Gas Networks, IEEE Trans.on Power Syst. PP (99) (2015) 1-10.

[7] C. Unsihuay-Vila, J. W. Marangon-Lima, a. C. Z. De Souza, I. J. PerezArriaga, P. P. Balestrassi, A model to long-term, multiarea, multistage, and integrated expansion planning of electricity and natural gas systems, IEEE Trans. on Power Syst. 25 (2) (2010) 1154-1168.

[8] S. A. S. An, Q. L. Q. Li, T. W. Gedra, Natural gas and electricity optimal power flow, 2003 IEEE PES T\&D Conference 1 (1) (2003) 138-143.

[9] C. Unsihuay, J. Lima, a. D. Souza, Modeling the Integrated Natural Gas and Electricity Optimal Power Flow, in: IEEE PES General Meeting, IEEE, 2007, pp. 1-7.

[10] M. Geidl, G. Andersson, Optimal power flow of multiple energy carriers, IEEE Trans. on Power Syst. 22 (1) (2007) 145-155.

[11] M. Chaudry, N. Jenkins, G. Strbac, Multi-time period combined gas and electricity network optimisation, Electric Power Systems Research 78 (7) (2008) 1265-1279.

[12] A. Quelhas, E. Gil, J. D. McCalley, S. M. Ryan, A Multiperiod Generalized Network Flow Model of the U.S. Integrated Energy System: Part IModel Description, IEEE Trans. on Power Syst. 22 (2) (2007) 829-836.

[13] C. Liu, M. Shahidehpour, Y. Fu, Z. Li, Security-Constrained Unit Commitment With Natural Gas Transmission Constraints, IEEE Trans. on Power Syst. 24 (3) (2009) 1523-1536.

[14] L. Bai, F. Li, H. Cui, T. Jiang, H. Sun, J. Zhu, Interval optimization based operating strategy for gas-electricity integrated energy systems considering demand response and wind uncertainty, Applied Energydoi:10.1016/j.apenergy.2015.10.119.

[15] P. Jirutitijaroen, S. Kim, O. Kittithreerapronchai, J. Prina, An optimization model for natural gas supply portfolios of a power generation company, Applied Energy 107 (2013) 1-9.

[16] X. Liu, J. Wu, N. Jenkins, A. Bagdanavicius, Combined analysis of electricity and heat networks, Applied Energy (2015) 1-13.

[17] P. Sorknæs, H. Lund, A. N. Andersen, Future power market and sustainable energy solutions The treatment of uncertainties in the daily operation of combined heat and power plants, Applied Energy 144 (2015) 129-138.

[18] X. Fang, Q. Hu, F. Li, B. Wang, Y. Li, Coupon-Based Demand Response Considering Wind Power Uncertainty : A Strategic Bidding Model for Load Serving Entities, IEEE Trans. on Power Syst. PP (99) (2015) 1-13.

[19] H. R. Mashhadi, S. Mohtashasmi, Economic Dispatch Problem Considering Natural Gas Transportation Cost, in: World Academy of Science, Vol. 38, 2009, pp. 1482-1487. 
[20] M. Qadrdan, J. Wu, N. Jenkins, J. Ekanayake, Operating strategies for a gb integrated gas and electricity network considering the uncertainty in wind power forecasts, IEEE Trans.on Sust. Energy 5 (1) (2014) 128-138.

[21] R. Rubio, D. Ojeda-Esteybar, O. Ano, A. Vargas, Integrated natural gas and electricity market: A survey of the state of the art in operation planning and market issues, 2008 IEEE/PES T\&D Conference and Exposition: Latin America.

[22] G. Strbac, Demand side management: Benefits and challenges, Energy Policy 36 (12) (2008) 4419-4426.

[23] C. Sahin, Z. Li, M. Shahidehpour, I. Erkmen, Impact of natural gas system on risk-constrained midterm hydrothermal scheduling, IEEE Trans. on Power Syst. 26 (2) (2011) 520-531.

[24] R. Z. Ríos-Mercado, C. Borraz-Sánchez, Optimization problems in natural gas transportation systems: A state-of-the-art review, Applied Energy 147 (2015) 536-555. doi:10.1016/j.apenergy.2015.03.017.

[25] PJM, Operational Analysis.

URL http://www.pjm.com/markets-and-operations/ops-analysis .aspx

[26] NYISO, NYISO Market Operations. URL http://www . nyiso.com/public/markets_operations/

[27] P. M. Coelho, C. Pinho, Considerations About Equations for Steady State Flow in Natural Gas Pipelines XXIX (3) (2007) 262-273.

[28] M. Mohitpour, H. Golshan, A. Murray, Pipeline Design \& Construction: A Practical Approach. 2000, American Society of Mechanical Engineers Press, New York.

[29] P. Palensky, D. Dietrich, Demand Side Management: Demand Response, Intelligent Energy Systems, and Smart Loads, IEEE Trans. on Industrial Informatics 7 (3) (2011) 381-388. doi:10.1109/TII.2011.2158841.

[30] F. Rahimi, A. Ipakchi, Demand response as a market resource under the smart grid paradigm, IEEE Trans. on Smart Grid 1 (1) (2010) 82-88. doi:10.1109/TSG.2010.2045906.

[31] Z. Li, Q. Guo, H. Sun, J. Wang, Storage-like devices in load leveling: Complementarity constraints and a new and exact relaxation method, Applied Energy 151 (2015) 13-22.

[32] S. A. Gabriel, A. J. Conejo, J. D. Fuller, B. F. Hobbs, C. Ruiz, Complementary models in energy markets, Springer, 2013.

\section{Appendix A. Linearization of the Product Term in (19)}

According to strong duality theorem, the objective function of the primal problem is equal to that of the dual problem at the optimum. The lower-level object (22) at each time step is given as:

$$
\begin{aligned}
& \sum_{i} \alpha_{i}^{S} G_{i}^{S}+\sum_{i} \alpha_{i}^{V} G_{i}^{V}+\sum_{i} \alpha_{i}^{O} G_{i}^{O} \\
= & \lambda \sum_{i} D_{i}-\sum_{i, \phi} \omega_{i, \max }^{\phi} G_{i, \max }^{\phi}+\sum_{i, \phi} \omega_{i, \min }^{\phi} G_{i, \min }^{\phi} \\
& +\sum_{l} \mu_{l}^{\min }\left(-C_{l}+\sum_{i} G S F_{l-i} \times D_{i}\right) \\
& +\sum_{l} \mu_{l}^{\max }\left(-C_{l}-\sum_{i} G S F_{l-i} \times D_{i}\right)
\end{aligned}
$$

With (28), the non-linear product term can be expanded as:

$$
\begin{aligned}
& \sum_{i} \pi_{i}\left(G_{i}^{S}+G_{i}^{V}\right) \\
= & \sum_{i} \lambda_{i}\left(G_{i}^{S}+G_{i}^{V}\right) \\
& +\sum_{i}\left[\left(G_{i}^{S}+G_{i}^{V}\right) \sum_{i} G S F_{l-i}\left(\mu_{l}^{\min }-\mu_{l}^{\max }\right)\right]
\end{aligned}
$$

From the equilibrium constraints (36)-(37) the following equivalents are obtained:

$$
\begin{aligned}
& \omega_{i, \text { min }}^{\phi} G_{i}^{\phi}=\omega_{i, \text { min }}^{\phi} G_{i, \text { min }}^{\phi} \\
& \omega_{i, \text { max }}^{\phi} G_{i}^{\phi}=\omega_{i, \text { max }}^{\phi} G_{i, \text { max }}^{\phi}
\end{aligned}
$$

Combining (35), (A.3) and (A.4) with (A.2) yields:

$$
\begin{aligned}
& \sum_{i, \varphi} \pi_{i} G_{i}^{\varphi} \\
= & \sum_{i, \varphi} \alpha_{i} G_{i}^{\varphi}-\sum_{i, \varphi}\left(\omega_{i, \min }^{\varphi} G_{i, \min }^{\varphi}-\omega_{i, \max }^{\varphi} G_{i, \max }^{\varphi}\right)
\end{aligned}
$$

Substituting (A.1) into (A.5) renders:

$$
\begin{aligned}
& \sum_{i, \varphi} \pi_{i} G_{i}^{\varphi} \\
= & \lambda \sum_{i} D_{i}-\sum_{i} \omega_{i, \max }^{O} G_{i, \max }^{O}+\sum_{i} \omega_{i, \min }^{O} G_{i, \min }^{O} \\
& +\sum_{l} \mu_{l}^{\min }\left(-C_{l}+\sum_{i} G S F_{l-i} \times D_{i}\right) \\
& +\sum_{l} \mu_{l}^{\max }\left(-C_{l}-\sum_{i} G S F_{l-i} \times D_{i}\right) \\
& -\sum_{i} \alpha_{i}^{O} G_{i}^{O} \\
\forall \varphi \in & \{S, V\}, \forall i \text { for }(A .5)-(A .6)
\end{aligned}
$$

\section{Appendix B. Linearization of Quadratic Costs}

The linearized cost function for generator $i$ at time $t$ is given by:

$$
\begin{aligned}
& W_{i, t}^{L}=\rho_{i} c_{i, 0}+\rho_{i} \sum_{\tau} K_{i, \tau} \delta_{i, t, \tau}, \\
& \forall i, \forall t, \forall \tau=1,2, . . N L_{i} \\
& G_{i, t}=G_{i, \min }+\sum_{\tau} \delta_{i, t, \tau} \\
& \delta_{i, t, 1} \leq T_{i, 1}-G_{i, \min } \\
& \delta_{i, t, \tau} \leq T_{i, \tau}-T_{i, \tau-1} \\
& \delta_{i, t, N L_{i}} \leq G_{i, \max }-T_{i, \tau-1} \\
& \delta_{i, t, \tau} \geq 0
\end{aligned}
$$

\section{Appendix C. Six-Bus System Parameters}

\begin{tabular}{|l||l|l|l|l|}
\hline Unit & $\begin{array}{l}\text { af (GJ/ } \\
\text { MW }^{2} \text { h) }\end{array}$ & $\begin{array}{l}\text { bf (GJ/ } \\
\text { MWh) }\end{array}$ & $\begin{array}{l}\text { cf }(\mathrm{GJ} / \\
\text { h) }\end{array}$ & $\begin{array}{l}\text { Gas } \\
\text { Price }(\$ / \\
\text { GJ) }\end{array}$ \\
\hline \hline G1 & 0.0011 & 47.46 & 179.28 & 6.57 \\
G2 & 0.0021 & 42.18 & 147.65 & 6.57 \\
G3 & 0.0004 & 58.00 & 186.61 & 6.57 \\
G4 & 0.0011 & 73.82 & 137.07 & 6.57 \\
G5 & 0.0053 & 68.55 & 144.91 & 6.57 \\
\hline
\end{tabular}

Table C.4: Heat Parameters of Generators 


\begin{tabular}{|l||l|l|l|l|}
\hline Unit & $\begin{array}{l}\text { Pmin } \\
(\mathrm{MW})\end{array}$ & $\begin{array}{l}\text { Pmax } \\
(\mathrm{MW})\end{array}$ & $\begin{array}{l}\mathrm{R} \\
(\mathrm{MW} / \mathrm{h})\end{array}$ & $\begin{array}{l}\text { Initial } \\
\text { Output } \\
(\mathrm{MW})\end{array}$ \\
\hline \hline G1 & 0 & 60 & 50 & 0 \\
G2 & 0 & 200 & 70 & 50 \\
G3 & 100 & 200 & 55 & 100 \\
G4 & 10 & 60 & 50 & 10 \\
G5 & 10 & 20 & 20 & 10 \\
\hline
\end{tabular}

\begin{tabular}{|c||c|c|c|}
\hline Index & From Node & To Node & $K_{m n}^{W}(\mathrm{Mcm} / \mathrm{KPa})$ \\
\hline \hline Pipe 1 & 1 & 2 & 9.87 \\
Pipe 2 & 2 & 5 & 7.32 \\
Pipe 3 & 5 & 6 & 8.84 \\
Pipe 4 & 3 & 5 & 8.49 \\
Pipe 5 & 4 & 7 & 9.78 \\
\hline
\end{tabular}

Table C.5: Electric Parameters of Generators

\begin{tabular}{|l||l|l|l|l|l|}
\hline Branch & $\begin{array}{l}\text { From } \\
\text { Bus }\end{array}$ & $\begin{array}{l}\text { To } \\
\text { Bus }\end{array}$ & $\begin{array}{l}\text { R } \\
\text { (p.u.) }\end{array}$ & $\begin{array}{l}\text { X } \\
\text { (p.u.) }\end{array}$ & $\begin{array}{l}\text { Flow } \\
\text { Limit } \\
\text { (MW) }\end{array}$ \\
\hline \hline Line 1 & 1 & 2 & 0.005 & 0.17 & 200 \\
Line 2 & 1 & 4 & 0.003 & 0.258 & 100 \\
Line 3 & 2 & 4 & 0.007 & 0.197 & 100 \\
Line 4 & 5 & 6 & 0.002 & 0.14 & 100 \\
TF 1 & 2 & 3 & 0 & 0.037 & 100 \\
TF 2 & 4 & 5 & 0 & 0.037 & 100 \\
PS 1 & 3 & 6 & 0.0005 & 0.018 & 100 \\
\hline
\end{tabular}

\begin{tabular}{|c|c|c|c|c|c|c|c|}
\hline Index & $\begin{array}{c}\text { Inlet } \\
\text { Node }\end{array}$ & $\begin{array}{c}\text { Outlet } \\
\text { Node }\end{array}$ & $\alpha$ & $K_{1}$ & $K_{2}$ & Rmin & Rmax \\
\hline \hline $\mathrm{C} 1$ & 4 & 2 & 0.25 & 0.123 & 0.075 & 1.6 & 2.45 \\
\hline
\end{tabular}

Table C.11: Parameters of Compressor

\begin{tabular}{|c|c|c|c|c|c|c|}
\hline Index & $\begin{array}{l}\text { af } \\
(\mathrm{GJ} / \\
M W^{2} h\end{array}$ & $\begin{array}{l}\text { bf } \\
(\mathrm{GJ} / \\
\mathrm{MWh})\end{array}$ & $\begin{array}{l}\text { cf } \\
(\mathrm{GJ} / \mathrm{h})\end{array}$ & Node & $\begin{array}{l}H_{\min } / \\
k W\end{array}$ & $\begin{array}{l}H_{\max } / \\
k W\end{array}$ \\
\hline \hline $\mathrm{C} 1$ & 0 & 0.22 & 52.73 & 2 & 298.28 & 447.42 \\
\hline
\end{tabular}

Table C.6: Transmission Network Parameters

Table C.12: Parameters of Compressor

\begin{tabular}{|l|l||l|l||l|l||l|l|}
\hline Hour & $\begin{array}{l}\text { Load } \\
\text { (MW) }\end{array}$ & Hour & $\begin{array}{l}\text { Load } \\
\text { (MW) }\end{array}$ & Hour & $\begin{array}{l}\text { Load } \\
\text { (MW) }\end{array}$ & Hour & $\begin{array}{l}\text { Load } \\
\text { (MW) }\end{array}$ \\
\hline \hline 1 & 175.19 & 7 & 173.39 & 13 & 242.18 & 19 & 245.97 \\
2 & 165.15 & 8 & 190.40 & 14 & 243.60 & 20 & 237.35 \\
3 & 158.67 & 9 & 205.56 & 15 & 248.86 & 21 & 237.31 \\
4 & 154.73 & 10 & 217.20 & 16 & 255.79 & 22 & 227.14 \\
5 & 155.06 & 11 & 228.61 & 17 & 256 & 23 & 201.05 \\
6 & 160.48 & 12 & 236.10 & 18 & 246.74 & 24 & 196.75 \\
\hline
\end{tabular}

\begin{tabular}{|c||c|c|c|}
\hline Supplier No. & Node No. & $\begin{array}{l}\text { Min Output } \\
(\mathrm{Mcm} / \mathrm{h})\end{array}$ & $\begin{array}{l}\text { Max Output } \\
(\mathrm{Mcm} / \mathrm{h})\end{array}$ \\
\hline \hline 1 & 7 & 65.14 & 150.10 \\
2 & 6 & 28.32 & 169.92 \\
\hline
\end{tabular}

Table C.13: Parameters of Natural gas well

Table C.7: Electricity Load Data of 24 hours

\begin{tabular}{|c||c|c|c|}
\hline Load & 1 & 2 & 3 \\
\hline \hline Bus No. & 3 & 4 & 5 \\
Factor & 0.4 & 0.3 & 0.3 \\
IBDR Price (\$/MWh) & 50 & 50 & 50 \\
\hline
\end{tabular}

\begin{tabular}{|c||c|c|}
\hline Load No. & Node No. & Distribution Factor \\
\hline \hline L1 & 1 & Gas consumption of $G_{1}$ \\
L2 & 2 & Gas consumption of $G_{2}$ \\
L3 & 1 & 2/3 of Residential gas load \\
L4 & 3 & 1/3 of Residential gas load \\
\hline
\end{tabular}

Table C.14: Parameters of Gas Load

Table C.8: Electric Load Distribution Factor

\begin{tabular}{|c||c|c|}
\hline Node No. & $\begin{array}{l}\text { Min Pressure } \\
(\mathrm{KPa})\end{array}$ & $\begin{array}{l}\text { Max Pressure } \\
(\mathrm{KPa})\end{array}$ \\
\hline \hline 1 & 724.0 & 1054 \\
2 & 956.3 & 1172 \\
3 & 1034 & 1344 \\
4 & 482.63 & 689.47 \\
5 & 1034 & 1379 \\
6 & 1103 & 1655 \\
7 & 689.47 & 956.3 \\
\hline
\end{tabular}

Table C.9: Parameters of Natural Gas Nodes

\begin{tabular}{|l|l||l|l||l|l||l|l|}
\hline Hour & $\begin{array}{l}\text { Gas } \\
\text { Load } \\
(\mathrm{Mcm} / \mathrm{h})\end{array}$ & Hour & $\begin{array}{l}\text { Gas } \\
\text { Load } \\
(\mathrm{Mcm} / \mathrm{h})\end{array}$ & Hour & $\begin{array}{l}\text { Gas } \\
\text { Load } \\
(\mathrm{Mcm} / \mathrm{h})\end{array}$ & $\begin{array}{l}\text { Gas } \\
\text { Houd } \\
(\mathrm{Mcm} / \mathrm{h})\end{array}$ \\
\hline \hline 1 & 147.83 & 7 & 158.03 & 13 & 169.92 & 19 & 185.21 \\
2 & 139.33 & 8 & 171.62 & 14 & 161.42 & 20 & 192.00 \\
3 & 132.53 & 9 & 175.02 & 15 & 163.12 & 21 & 188.61 \\
4 & 134.24 & 10 & 179.55 & 16 & 166.52 & 22 & 185.21 \\
5 & 116.11 & 11 & 173.32 & 17 & 171.62 & 23 & 171.62 \\
6 & 159.70 & 12 & 173.32 & 18 & 176.72 & 24 & 156.33 \\
\hline
\end{tabular}

Table C.15: Residential Gas Load Data of 24 hours 


\section{Nomenclature}

\section{Constants}

$\alpha_{i}^{O} \quad$ Estimated bidding price of non-strategic generator on bus $i$

$\rho_{i}^{G A S} \quad$ Unit price of natural gas at node $i$

$C_{l} \quad$ Power flow limit on transmission line $l$

$c_{i, 0} \quad$ Gas consumption constant coefficient of CCGT unit $i$

$c_{i, 1} \quad$ Gas consumption linear coefficient of CCGT unit $i$

$D_{i, t} \quad$ Electric demand on bus $i$ at time $t$

$d_{i, t}^{\max } \quad$ Maximum demand of gas load $i$ at time $t$

$d_{i, t}^{\text {min }} \quad$ Minimum demand of gas load $i$ at time $t$

$F_{c} \quad$ Daily natural gas supply limit

$G_{i, \text { max }}^{O} \quad$ Upper power limit of non-strategic generator on bus $i$ at time $t$

$G_{i, \max }^{S} \quad$ Upper power limit of strategic generator $i$

$G_{i, \max }^{V}$ Upper power limit of virtual generator on $i$ at time $t$

$G_{i, \text { min }}^{O} \quad$ Lower power limit of non-strategic generator on bus $i$ at time $t$

$G_{i, \min }^{S} \quad$ Lower power limit of strategic generator $i$

$K_{m n}^{W} \quad$ Weymouth factor of pipeline $m n$

$L_{j} \quad$ Number of hours VPP $j$ must stay offline due to previous states

$M D_{j} \quad$ Minimum off-line time limit of VPP $j$

$M U_{j} \quad$ Maximum on-line time limit of VPP $j$

$p_{n, \max }$ Maximum natural gas pressure of node $n$

$p_{n, \min } \quad$ Minimum natural gas pressure of node $n$

$R_{i}^{D N} \quad$ Ramp down limit of generator on bus $i$

$R_{i}^{U P} \quad$ Ramp up limit of generator on bus $i$

$R_{j, \max }$ Maximum compression ratio of compressor $j$

$R_{j, \min } \quad$ Minimum compression ratio of compressor $j$

$s_{i, t}^{\max } \quad$ Maximum supply output of well $i$ at time $t$

$s_{i, t}^{\min } \quad$ Minimum supply output of well $i$ at time $t$

$T \quad$ Daily scheduling time horizon from 1 to 24

$G S F_{l-i}$ Generation shift factor from bus $i$ to line $l$

\section{Sets}

$A(j) \quad$ Set of Nodes connected to node $j$

$I_{c c g t}^{N G} \quad$ Set of buses having CCGT units

$I_{\text {load }}^{N G} \quad$ Set of nodes having natural gas load, including CCGT units

$I_{s p}^{N G} \quad$ Set of nodes having natural gas well

$I_{\text {comp }}^{N G} \quad$ Set of natural gas compressors

$I_{\text {comp }}^{N G}(j)$ Set of terminal nodes of compressor $j$

$J \quad$ Set of demand response based virtual power plants

$N \quad$ Set of natural gas nodes

$A_{\text {load }}^{N G} \quad$ Incidence matrix between natural gas loads and gas nodes
$A_{s p}^{N G} \quad$ Incidence matrix between natural gas wells and gas nodes

Units

GJ Gigajoules

$\mathrm{KPa}$ Kilopascal

Mcm Thousand cubic meters

\section{Variables}

$\alpha_{i}^{S} \quad$ Bidding price for strategic generator on bus $i$

$\alpha_{i}^{V} \quad$ Bidding price for strategic virtual power plant on bus $i$

$\lambda_{t} \quad$ Dual variable associated with network power balance

$\mu_{l, t}^{\max } \quad$ Dual variables associated with line $l$ flow upper limit

$\mu_{l, t}^{\min } \quad$ Dual variables associated with line $l$ flow lower limit

$\omega_{i, t, \max }^{O}$ Dual variables associated with upper limit of nonstrategic generator on bus $i$ at time $t$

$\omega_{i, t, \min }^{O}$ Dual variables associated with lower limit of nonstrategic generator on bus $i$ at time $t$

$\omega_{i, t, \max }^{S}$ Dual variables associated with upper limit of strategic generator on bus $i$ at time $t$

$\omega_{i, t \text { min }}^{S}$ Dual variables associated with lower limit of strategic generator on bus $i$ at time $t$

$\omega_{i, t, \max }^{V}$ Dual variables associated with upper limit of virtual power plant on bus $i$ at time $t$

$\omega_{i, t, \min }^{V}$ Dual variables associated with lower limit of virtual power plant on bus $i$ at time $t$

$\pi_{i, t} \quad$ Locational marginal price of bus $i$ at time $t$

$d_{i, t} \quad$ Gas demand of gas load $i$ at time $t$

$d_{i, t} \quad$ Gas demand on gas node $i$ at time $t$

$f_{c, j, t} \quad$ Natural gas flow through compressor $j$ at time $t$

$F_{i, t} \quad$ Fuel consumption of CCGT unit $i$ at time $t$

$G_{i, t} \quad$ Power output of CCGT unit $i$ at time $t$

$G_{i, t}^{O} \quad$ Power output of non-strategic generator on bus $i$

$G_{i, t}^{S} \quad$ Power output of strategic generators

$G_{i, t}^{V} \quad$ Power output of virtual power plants

$H_{j, t} \quad$ Power consumption of compressor $j$ at time $t$

$p_{n} \quad$ Nodal pressure of node $n$

$p_{m n}^{i n} \quad$ Nodal pressure on the inlet node of pipeline $m n$

$p_{m n}^{\text {out }} \quad$ Nodal pressure on the outlet node of pipeline $m n$

$R_{j, t} \quad$ Compression ratio of compressor $j$ at time $t$

$s_{i, t} \quad$ Gas output of gas well $i$ at time $t$

$v_{j, t} \quad$ Power output of virtual power plant $j$ at time $t$

$14 W_{i, t} \quad$ Natural gas cost of CCGT unit $i$ at time $t$

$W M_{m n}$ Gas flow in pipeline $m n$

$p_{n, t} \quad$ Gas imbalance on node $n$ at time $t$ 